\title{
Scaffolding RAMI4.0-Exploration as Design Support
}

\author{
Claudia Kaar \\ Johannes Kepler University \\ Institute of Business Informatics - \\ Communications Engineering \\ Linz, Austria \\ claudia.kaar@jku.at
}

\author{
Josef Frysak \\ Johannes Kepler University \\ Institute of Business Informatics - \\ Communications Engineering \\ Linz, Austria \\ josef.frysak@jku.at
}

\author{
Christian Stary \\ Johannes Kepler University \\ Institute of Business Informatics - \\ Communications Engineering \\ Linz, Austria \\ christian.stary@jku.at
}

\begin{abstract}
Developing Industry 4.0 applications is a complex endeavor, as it requires adjusting several relevant perspectives and items throughout design. RAMI4.0 has been introduced to facilitate Industry 4.0 developments. It puts items from three different development dimensions in mutual context. Some items are standardized due to existing technical standards, business-oriented ones depend on the development case at hand. Case-sensitive adjustment includes resolving inconsistencies and overcoming coherence deficits. We propose scaffolding to structure and thus, facilitate design of Industry 4.0 applications based on RAMI4.0. It visualizes static and dynamic development entities for guiding the connection of RAMI4.0 elements on various levels of granularity. We exemplify our approach using a common Industry 4.0 demonstrator, "MyJoghurt". It provides evidence of supportive guidance for developers when exploring RAMI4.0 through engaging scaffolds.
\end{abstract}

\section{KEYWORDS}

RAMI4.0, Industry 4.0, Digital Architecting, Exploration, MyJoghurt, Scaffolding

\section{ACM Reference Format:}

Claudia Kaar, Josef Frysak, and Christian Stary. 2018. Scaffolding RAMI4.0-Exploration as Design Support. In ECCE'18: Proceedings of the 36th European Conference on Cognitive Ergonomics (ECCE2018), September 5-7, 2018, Utrecht, Netherlands. ACM, New York, NY, USA, 8 pages. https://doi.org/10.1145/3232078.3232098

\section{INTRODUCTION}

When organizations shift to Industry 4.0 (I4.0) conform structures, networked communication enables digital production

\footnotetext{
Permission to make digital or hard copies of part or all of this work for personal or classroom use is granted without fee provided that copies are not made or distributed for profit or commercial advantage and that copies bear this notice and the full citation on the first page. Copyrights for thirdparty components of this work must be honored. For all other uses, contact the owner/author(s).

ECCE'18, September 5-7, 2018, Utrecht, Netherlands

(c) 2018 Copyright held by the owner/author(s).

ACM ISBN 978-1-4503-6449-2/18/09.

https://doi.org/10.1145/3232078.3232098
}

processes supported by electronic equipment and automation. New business models emerge through the communication between products, machines, and organizations for the benefit of companies and customers [10]. In I4.0, horizontal and vertical integration of information and communication flows (i.e., processes) within and across organizations are essential, as well as thorough digitization in engineering $[12,19]$. In order to transform organizations to that respect, standards and enabling technologies are developed.

The Reference Architecture Model 4.0 (RAMI4.0) has been developed by a committee (composed of technical experts in the area of I4.0 and cyber-physical systems) of the VDI/VDE Society of Measurement [6]. Following an approach similar to smart grids, RAMI4.0 introduces a three-dimensional model. It comprises six architectural interoperability layers ranging from Asset to Business, seven Hierarchy Levels based on the automation pyramid, and the Life Cycle \& Value Stream of systems and products involved in industrial production processes.

To gain a deeper understanding of RAMI4.0 and to explore different areas within the framework, a fragmentation of each dimension could be helpful. By separating it into building blocks, the scope of each element can be made transparent. However, differing understanding of terms and concepts makes the exploration a challenging task. Designers need to spend severe effort to integrate existing knowledge and standards from different fields to model appropriate I4.0 applications.

In this paper we introduce scaffolding RAMI4.0 explorations for effective I4.0 application design. The approach visualizes essential items of RAMI4.0 and their connections, thereby facilitating coherent and consistent knowledge integration. We demonstrate an approach by applying RAMI4.0 on a commonly accepted sample case called "MyJoghurt". In Section 2, we introduce RAMI4.0 and detail the I4.0 demonstration case. In Section 3, the scaffolding concept is introduced. We also argue why and how scaffolds should be used in our context. In Section 4, we introduce Structural Scaffolds for RAMI4.0, as they provide the basis for procedural guidance exemplified in Section 5. Results are shown in Section 6. Section 7 and 8 conclude the paper. 


\section{BACKGROUND}

RAMI4.0 [21] has been introduced to structure the development of I4.0 applications into manageable parts including rules for implementation. We detail RAMI4.0 and the "MyJogurt" demonstrator case in the following.

\section{RAMI4.0}

RAMI4.0 was originally introduced by the Plattform Industrie 4.0 in 2015 and was designed to facilitate interrelating and detailing I4.0 concepts [16]. It was created by a group of technical experts in the area of I4.0 and cyber-physical systems of the VID/VDE Society of Measurement [21]. Based on the Smart Grid Architecture Model (SGAM) [2], RAMI4.0 constitutes an architecture as frame of reference for I4.0 application development. RAMI4.0 is a three-dimensional model with goals, e.g., to be a simple and manageable architecture model, identification of reference, and closure of gaps for existing standards, and to identify use case contents and relationships [21]. RAMI4.0 is a three dimensional model which is divided into architectural Layers, Hierarchy Levels, and Life Cycle \& Value Stream. Fig. 1 shows the model and will be explained as in [7]:

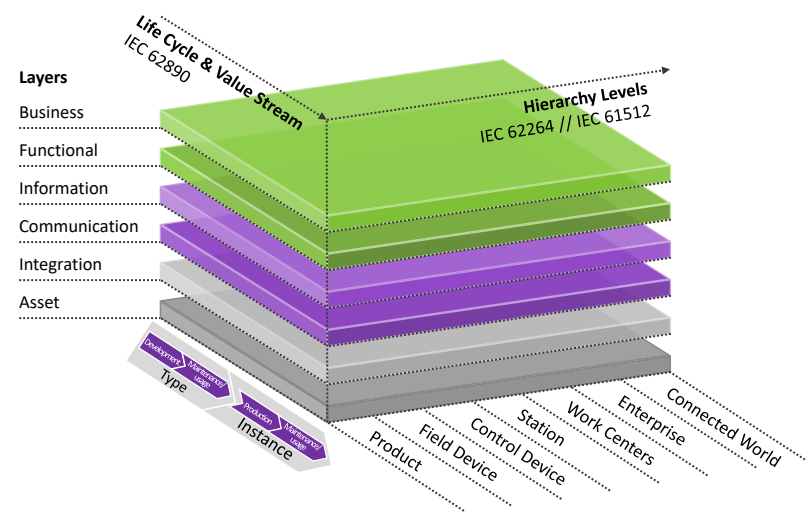

Figure 1: RAMI4.0 (according to [21, p. 7]).

The Layers in RAMI 4.0 are separated into the Business, Functional, Information, Communication, Integration, and Asset Layer. These architectural Layers are derived from SGAM and are refined by splitting the Component Layer of SGAM into two separate layers, Integration and Asset. Hereby, the goal was to detach the physical from the immaterial information world. Starting from the bottom, the Asset Layer represents the physical object itself. It characterizes the reality, e.g., metal parts, documents, even humans can be part of this layer. Through the Integration Layer, non communicating assets can be involved in the information world. By representing the digital twin of the asset, the Integration Layers' main goal is to provide the information about the asset and makes it computer-processable. Furthermore, this layer can generate events from the asset and gives control over technical processes. The Communication Layer handles the communication between the assets and implementation of processes. The most common standard on this layer is OPC UA (IEC 62541) which is based on the ISO-OSI layered communication protocol model (ISO/IEC 7498-1:1994). The model serves as overview and describes the layer on a more precise level. The Information Layer is probably the most advanced layer in respect of the known standards. It provides the run time environment for processing events, and executes and contains the formal description of rules. Furthermore, this layer persists the data of the models, assures data integrity, integrates the different data consistently, and provides structured data via service interfaces. The Functional Layer generates rules and some decision making logic, formally describes functions, serves as the platform for horizontal integration of functions, and is the run time and modeling environment for services which support business processes. Finally, the Business Layer ensures the integrity of the functions in the Value Stream, maps the business models to the overall processes, contains legal and regulatory framework conditions, and orchestrates the services of the Functional Layer.

The Hierarchy Levels serve as vertical integration within a factory or plant. Based on the automation pyramid [2], two more levels were added, i.e., Product and Connected World. The Product was added because it will take a crucial role in the process and therefore is an essential part of I4.0. Moreover, the level Connected World was added to show the connection and interrelation across enterprise boundaries. As can be seen in Fig. 2 the levels do not need to communicate with the ones next to each other, they are able to connect with any level they need to, and thus form a network.

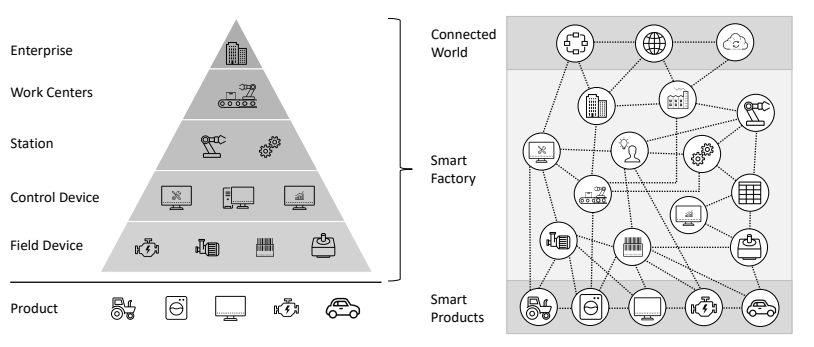

Figure 2: Hierarchy (according to $[7$, p. 45]).

The Life Cycle \& Value Stream is divided into Type and Instance. The Type represents the idea of the product, beginning with development, including design, testing, and prototyping. After finishing all tests the product is released for production and moves to Instance, which represents the 
manufactured product. Both stages are separated into Development or Production, and Maintenance/Usage to gain an even deeper classification of the asset.

\subsection{Demonstrator}

In order to facilitate I4.0 application development, several Demonstrators are developed. In this paper, we use the I4.0 Demonstrator "MyJoghurt" $[14,15]$. It implements an agentbased network for cyber-physical systems. When producing yogurt throughout different locations, the agent of each plant can communicate with a virtual broker, which distributes the orders in an optimal ratio. Each plant specializes in a different process of the yogurt production. The main party is the order handler, which receives the orders and is the supplier of the customer. This scenario helps to exemplify different applications in all dimensions of RAMI4.0. The communication across and within enterprise boundaries can be identified. The agent-system depicts a network of the different production sites and therefore represents a connected world, and the production of the yogurt itself can be located on the product level.

\section{SCAFFOLDING}

In this section we introduce the concept and its capabilities to be utilized for conveying the meaning and application of complex frameworks, such as RAMI4.0. We start with explaining the concept of scaffolding and continue with its proof of concept for complex learning tasks, before providing some application guidelines stemming from experiental designs.

\section{Concept}

Scaffolding has been introduced to support a learner's selfexploratory capacity building capability when accomplishing complex learning tasks by adapting instructional interventions to his/her background, level of understanding, and competencies. Along the learning process, support is provided on 'how' and 'why' to perform a task while engaging with key disciplinary frameworks [9]. Support is gradually removed according to the learner's development of a learning strategy when aiming to achieve a specific learning goal. Originally identified by Vygotsky [22] when developing his socialcultural theory, scaffolding refers to the socio-cognitive field, more specifically to the zone of proximal development. He defined it as "the distance between the actual development level as determined by independent problem-solving and the level of potential development through problem solving under adult guidance or in collaboration with more capable peers" [22, p. 86]. In this zone, individuals build capacity "... to solve a problem, carryout a task, or achieve a goal, which would be beyond his (or her) unassisted efforts" [23, p. 90].
Although this process is understood to be an accompanied procedure supervised by a more experienced person, its concepts have propagated to digital learning support systems with corresponding guidance and monitoring features (cf. $[4,5])$.

\section{Complex Learning Task Support}

Scaffolding is of essential means to explore knowledge spaces in a guided, however, self-managed way. It enables learners capacity-building even in complex settings when aiming to solve a specific problem. Thereby, learners receive adapted guidance and assistance, helping them to complete a task independently, as shown in [13] for scaffolded knowledge integration in network learning. The process behind scaffolding has been investigated by Barnsky [1], showing benefits of combining learning with media-rich illustrations. Chen [3] also investigated further factors and could demonstrate that adaptive scaffolding appeals to learners and promotes improved performances and motivational outcomes. Specifically, the study suggests that learners with lower levels of knowledge who possessed extrinsic motivation benefited the most from the adaptive scaffolds.

As scaffolding instruments cannot only guide learners through task complexities, they may also trigger other important aspects, in particular, engaging learners in working with key disciplinary frameworks and strategies, going beyond technical skill development [18]. Such scaffolds can act as cognitive attractors. They can also foster elaborated task accomplishment in line with the learning goals. Thereby, learning processes take into account explanatory knowledge required for decision taking, and meta-cognition, such as reflecting on achieved results and progress. Both can be part of cognitive apprenticeship activities, when learners become increasingly experienced problem solvers.

For digital learning or exploration support, the guidance knowledge from mentors needs to be encoded. It then can be used for scaffolding learners through coaching, task structuring, and hints in digital environments, without explicitly providing final answers [17]. Scaffolding is supposed to support learning of both, how to accomplish a learning task and why a learning task should be accomplished in a certain way [8].

\section{Application Guidelines}

Chen [3] advised that cognitive and motivational scaffolds should be provided by a learning support system allowing learners to monitor their own achievements. Hence, they should be able to experience the results of their problemsolving activities and learning steps. In addition, formative assessments could be added to the system in order to stimulate the interactions between the materials and the learner. 
Finally, the study suggests that promoting positive learning outcomes requires taking into account students' prior knowledge. Since prior knowledge can have a significant impact on learning processes and outcomes when designing scaffolds, offering appropriate support for each individual determines whether learners actually can improve their performance. Hence, scaffolds for stakeholders with lower levels of prior knowledge should be dynamic in order to best assist them to build capacity. Hmelo-Silver et al. [9] could extract several principles that can be used when developing scaffolds for RAMI4.0 exploration. We have applied them when developing the scaffolds introduced in the next section.

Scaffolds should make explicit disciplinary thinking and strategies: Since RAMI4.0 is a multi-dimensional framework triggered by business, organizational, and technological factors, the background of each asset or item should be made transparent and accessible. In this way, the various elements that need to be aligned in the course of development can be justified from an operational and strategic perspective.

Scaffolds should embed expert guidance: RAMI4.0 can be considered a framework of expertise from various disciplines. Its elements capture some expert knowledge that have been either standardized or encoded in a way enabling their digital processing. For completing a development task, each RAMI4.0 element can contribute expert knowledge for solving complex problems.

Scaffold should structure complex tasks to reduce cognitive level: RAMI4.0 is a fine-grained approach along three axes, which in turn allow decomposing a development task according to a triple descriptor. However, the effort required to 'step-through' the multi-dimensional framework and selecting relevant RAMI4.0 elements for solving a case at hand can be substantial.

\section{SCAFFOLDING RAMI4.0-EXPLORATION}

In this section we detail the proposed scaffolds and exemplify their handling using the I4.0 Demonstrator case. In the first subsection, Structural Scaffolds are detailed, and in the second subsection the Procedural ones, both of which are suitable for supporting development processes.

\section{Structural Scaffolds}

We consider Structural Scaffolds to enhance the framework structure independent of handling its elements. In the following we detail each of the proposed Structural Scaffolds: (i) RAMI 4.0 Building Blocks, (ii) Building Block Rooms, (iii) Building Block Room's Interior. Fig. 3 shows the first Structural Scaffold. It decomposes the cube into 3D-elements, socalled RAMI4.0 Building Blocks. They represent mutually related design items that need to be aligned in the course of development. In contrast to the original structure, relations between elements can be named in order to provide relevant context information. The relations can be omitted in case of advanced RAMI4.0 understanding. Fig. 4 shows the second Structural Scaffold. It corresponds to zooming into RAMI4.0 Building Blocks and uses the room metaphor: Building Block Rooms. Each 3D-element has doors to enter and leave the room. It also contains a facility to go down to the next element or to move through the ceiling, since an element can be related to design elements in all directions in the course of development. This facility can be removed for advanced learning support.

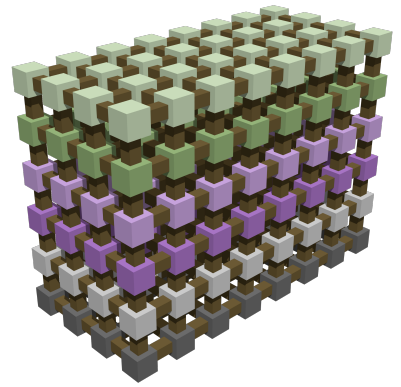

Figure 3: Structural Scaffold I: Decomposing RAMI4.0 into mutually connected Building Blocks.

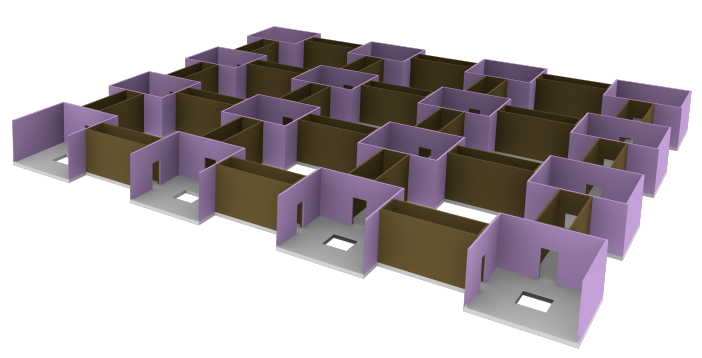

Figure 4: Structural Scaffold II: Building Block Rooms.

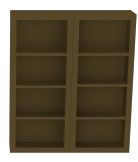

Figure 5: Structural Scaffold III: Item Storage.

Fig. 5 shows the third Structural Scaffold. It is the interior of a RAMI4.0 Building Block and uses the storage metaphor. It is termed Item Storage. Each element of the framework has not only doors to enter and leave the room, but also a storage device to keep and locate development items, e.g., information model elements such as business rules. They can be predefined through standards or need to be added to the case at hand. Storage devices can also be left empty in case of informed developers. 


\section{Procedural Scaffolds}

Since Procedural Scaffolds deal with utilizing RAMI4.0 elements, we introduce them along a "MyJoghurt" process as an exemplary networked production. As already indicated, various types of development items, including processes, production plants or parts of them, software components, technologies and standards, or people and organizations representing stakeholders, can be elements and thus, design items [20]. For RAMI4.0-conform development, they become part of processes allocated to the three dimensions of RAMI4.0 and their elements including possible standards, which can be used to implement this process.

Typically, stakeholder or role-relevant information is positioned on the first three Layers of RAMI4.0. The Business Layer illustrates the basic process model used for communication, e.g., through message passing. Since the Business Layer does not include a standard to model business processes, companies can decide which approach to use. They capture the interaction behavior of the involved stakeholders, namely the customer, the yogurt supplier, and production plant, and thus, can be captured by a Procedural Scaffold.

The Functional Layer typically contains all functions and services needed to execute the business process. Similar to the Business Layer, this layer does not define any specific standards except for specific types of functions. However, three different functions are defined, i.e., basic functions, process functions, and manufacturer-specific functions [7, p 51f]. Basic functions are general, I4.0 compliant functions such as "condition monitoring". As the communication in the use case crosses enterprise boundaries, the I4.0-compliant format and semantics in the Information Layer need to be used. Without collaboration, services, e.g., diagnosis or monitoring, cannot be provided. Process functions include standardized steps in a production process, such as those defined in DIN 8580. Therefore, each individual production plant needs to consider already available standards for its machines. Manufacturer-specific functions are likely to be different for each manufacturer, and will be indicated by a Procedural Scaffold.

The Information Layer contains the information model for the business process. Several standards are already available to define the different models. For example, the IEC Common Data Dictionary, or characteristics, classification, and tools of eCl@ss are recommended to be used on this level. The important key issue here is to define a single information model and its syntax and semantics which are validated and accepted throughout the company and, in this case, across enterprise boundaries. Finally, the Communication Layer defines a standard to be used for communication, OPC UA (IEC 62541). Elements of these layers need to be selected when executing Procedural Scaffolds in the Demonstrator case.

\section{APPLICATION OF SCAFFOLDS}

In this section we demonstrate the use of the scaffolds for the Information Layer and a cross-cutting application from the Functional to Information Layer.

\section{Example: Information Layer}

The first example shows the application of scaffolds in the Information Layer. Within that Layer from Type/Instance perspective, development Planning for time of production needs to be specified on the RAMI4.0 level Product. Fig. 6 shows the relevant part of the Information Layer. On the left side the identified RAMI4.0 element is a Rule. It contains formal description which need to be executed to achieve production results and is located in the Room Product (exemplified for milk production):

- For the RAMI4.0 Building Block Product: The rate of lactic acid has to be $0.7-1.1 \%$, to be produced with this Field Device.

- For the linked RAMI4.0 Building Block Field Device: The machine must be able to the heat the Product (milk) to 37 degrees Celsius at a minimum, but not exceeding 45 degrees of Celsius.

On the Instance side of Type/Instance axis, still on the RAMI4.0 Information Layer, the execution of the planning of production is linked to the Type Building Block Field Device above:

- For the executing RAMI4.0 Building Block Field Device: When the RAMI 4.0 element Rule is executed on this Field Device, the temperature is persisted in a log in OpenPLC (binary) format on a flash device.

Fig. 6 shows Procedural Scaffold I (Navigating through Rooms) allowing to represent I4.0 development knowledge. As shown in this example, it enables directly linking RAMI4.0 Building Block Type along the Type/Instance axis to the Building Block Instance. In this way, developers are able to explore the move from planning to execution of a production step, e.g., each rule needs to be implemented in order to become effective in production.

The procedural relation is encoded by a directed visual link starting from a specific Building Block Room (i.e. a Structural Scaffold) to another Room (see Fig. 6) from the left Room to the right one. However, the Room addressed by a link does not necessarily have to be located on the same axis, layer, or level of RAMI4.0. The next example shows a crossing-cutting through different Layers. 


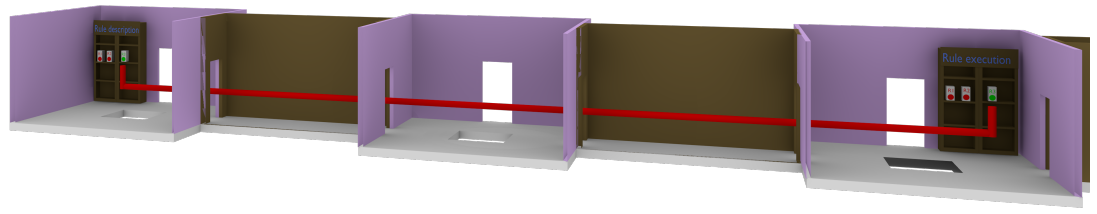

Figure 6: Procedural Scaffold I: Navigation through Rooms.

\section{Example: Cross-cutting of Layers}

To enhance the example given before, executing Rules on the Information Layer requires some services from the RAMI4.0 Functional Layer. In such a case, the Trap Door of the corresponding Room of the Functional Layer needs to be opened by using a Procedural Scaffold I. Again, it may require the Structural Scaffold II as learning support, as the Room has to be equipped with the proper exploration facilities. In this case, the Item Storage of the Functional Layer consists of the formal description of functions. Since a business process can access defined functions for their activities, we can define functions to handle the specific rule execution. By cross-cutting the layer from the Functional Layer to the Information Layer, the defined function accesses the specific rule, defined in the Information Layer, by opening the Trap Door, as defined in the Procedural Scaffold I.

As can be seen in the example, the Procedural Scaffold I helps identifying the link between the different Building Blocks. Moreover, links need not to be within a single layer, they can also cut through layers and Rooms. In addition, Procedural Scaffold II helps identifying the interior of Building Blocks and allows the arrangement of elements, such as Rules, in a context-sensitive while still structured way. Like Procedural Scaffold I, it can be stepwise removed in case developers need less learning support when exploring RAMI4.0.

\section{Ontological Context Information}

In order to provide more complex relationships an additional Procedural Scaffold has been developed: the Ontological Context Information. Fig. 7 shows such a scaffold that can be activated whenever a developer is located in one of the RAMI4.0 Building Block Rooms. It provides a concept map containing all ontological terms relevant for a certain Room. The map depicted in Fig. 7 allows entering the Rule concept which has a certain meaning according to the RAMI4.0 framework. By using concept maps a developer can be supported to grasp the meaning of RAMI4.0 terms and relations when designing an I4.0 application, in particular to get familiar with the inherent relations encoded between the various RAMI4.0 elements. As such, this scaffold complements a development case with all conceptual background knowledge available for RAMI4.0 elements. The development of the Ontological
Context Information structure underlying the Procedural Scaffold II has been detailed in [11].

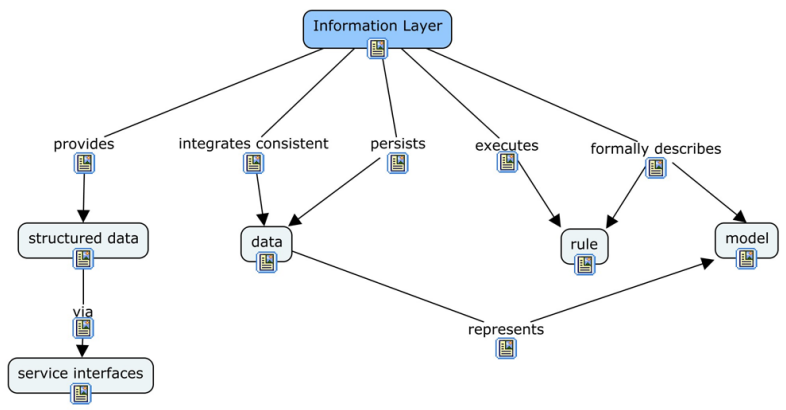

Figure 7: Procedural Scaffold II: Ontological Context Information $([11$, p. 91]).

Fig. 7 contains baseline information that has been used for the creation of the concept map representing the Information Layer. It originates from the standard document on DIN SPEC 91345. The part displayed in the scaffold in Fig. 7 shows six different functions of the Information Layer, e.g., provision of structured data via service interfaces, consistent integration and persistence of data, execution of rules, and the formal description of rules and models. The key concepts and the mutual links were enriched with the documentation and occurrence of the used term and can be accessed via links. This feature enables the developer to quickly identify and scan the source of a concept. By following the links and the used arrows a sentence can be built describing the functionality as defined. For example, the Information Layer provides structured data via service interfaces.

The map enables the developer to quickly understand the ideas of the different Layers and concepts within. It helps visualizing the connections between terms and ideas concerning RAMI4.0. Due to the different Layers, terms can be used in several ways and have different meaning on each individual level. Therefore, concept maps also capture the links to sources and standards, for direct reference to the concept at hand. Due to the aforementioned capabilities of concept maps for associating semantic information elements and dynamic navigation through different Layers, this scaffold can be used also along the other direction as introduced above. Once a developer decides to try understanding RAMI4.0 from 
a conceptual perspective before applying it in a particular context, he/she might follow the links from definitions and other sources to the respective Building Block in RAMI4.0. The represented concepts can be explored in parallel to develop further I4.0 use cases. Overall, the Procedural Scaffold II can be set according to the current understanding of a developer of RAMI4.0 elements and their relations.

\section{SUMMARY OF FINDINGS}

According to RAMI4.0, application-relevant knowledge, and thus design specifications do not only refer to a single Layer. Once several layers are affected, the navigation concept provided by the Procedural Scaffold I becomes essential. It needs to work in several directions. For instance, the Business Layer captures the operational processes that need to be supported, the Information Layer details the data and rules which need to be considered when executing the processes, while the Functional Layer captures required services to accomplish business objectives according to the specified processes. The scaffold can then be applied in the context of a specific development task, like handling the exemplified Rule as focus on the Information Layer. The layer represents a particular development aspect which at some point in time requires context information from other Layers. Then, the Procedural Scaffold II provides a backlink to another RAMI4.0 element of that Layer. The relationship to the other Layers have been encoded according to RAMI4.0 as links in the concept map (see [11]).

Overall, application development according to I4.0 requirements is driven by the vertical and horizontal integration of RAMI4.0 elements. Basically, an application development process can be structured following each of the RAMI4.0 axes: The separation of Types and Instances according to the Life Cycle \& Value Stream axis could be a starting point for facilitating design specification. The same holds for the RAMI4.0 Hierarchy Levels starting with a product perspective that propagates along various aspects to distributed production settings. However, once it comes to the RAMI4.0 Layers, developers are challenged. Then, process-relevant knowledge needs to be allocated to a specific RAMI4.0 Layer element. Thereby, Procedural Scaffold I is an effective orthogonal means to support the identification of relevant RAMI4.0 elements. In combination with the Procedural, Room-sensitive, Scaffold II (Ontological Context Information) it facilitates information allocation when both, procedural progressions along the Life Cycle axis and digital representation of objects, have to be allocated in a RAMI4.0-conform way.

The Procedural Scaffolds are of particular use when relating RAMI4.0 elements cross layers. Consider a specific process that is decomposed from an abstract description into smaller processes. Then, the complete Life Cycle \& Value Stream has to be followed - sub-processes and associated components are specified. Once they have been identified, they also need to be assigned to elements of the Layer and Hierarchy axes. Cutting across the Layers and Levels allows concurrently recognizing important related processes that need to be executed in parallel, e.g., for quality assurance or optimization.

\section{DISCUSSION}

Scaffolding has been introduced to facilitate developing I4.0 applications based on RAMI4.0. They support its exploration when adjusting its three inherent perspectives with development issues of a case at hand. Although RAMI4.0 has been introduced to facilitate I4.0 developments, its complexity challenges developers from a structural and procedural point of view. Since the entry point into the multidimensional structure is not prescribed by a development process specification, scaffolding is the proper means to be case-sensitive for development while providing orientation and guidance for utilizing RAMI4.0.

We have introduced three Structural Scaffolds and two Procedural ones that visualize static and dynamic development entities when exploring RAMI4.0. The Structural Scaffolds concern the framework structure as it is and implement zooming into a RAMI4.0 element. Starting with a view on the 3D-structure of the framework it breaks down elementand relation-specific information. It is followed by a Room scaffold that can be entered and left in many directions, and by an Item Storage scaffold trying to support organizing all information relevant in a particular Room. The Procedural Scaffolds depend on how the framework is actually used for developing an I4.0 application. Basically, they link Rooms and provide ontological background information to inform the next step for entering a Room.

Throughout the development process of the scaffolds we could follow Hmelo-Silver et al.'s [9] three major design principles: (i) Each scaffold makes explicit disciplinary thinking and strategies. All Structural Scaffolds allow focusing on axis-relevant information related to business, organizational, and technological factors, while making context available in a static form. All Procedural Scaffolds focus on linking disciplinary thinking as they focus on coupling elements to explicit context elements or information. (ii) Each scaffold embeds expert knowledge and guidance. Based on the background of RAMI4.0 and the practical experience with the Demonstrator case, each of the scaffold has been designed on the basis of existing information (structures) and practical experiences. (iii) Each scaffold structures a complex task to reduce the cognitive burden. The Structural Scaffolds are stepwise RAMI4.0 refinements, reducing complexity by stepby-step zooming in, where the Procedural Scaffolds are based on one-by-one relations. 


\section{CONCLUSION}

Although we could exemplify our approach and its potential using the reference I4.0 Demonstrator "MyJoghurt", further research needs to be done with respect to the Room metaphor. Currently it is not clear how relations cross-cutting Layers, Levels, and Values Streams can be visualized effectively. Another research concern is the Ontological Context Information scaffold. The underlying RAMI4.0 ontology is some kind of parallel information space that needs to be coupled to the RAMI4.0 elements in a coherent and transparent way. Finally, all social aspects concerning development, e.g., involving several experts need to be investigated, in order to structure development needs, and to adjust scaffolding procedures.

\section{ACKNOWLEDGMENT}

This work is partially funded by the Austrian Science Foundation (Upper Austrian Programme AS 2017 Digitalization, Contract no. 862006) provided for the research project SICHTEN 4.0 Sichtenorientiertes Engineering Cyber-Physischer Systeme mittels Existierender Normen der Industrie 4.0.

\section{REFERENCES}

[1] J. Barnsky. 2004. Connect the Dots of Management and Get "Unstuck". Quality Systems Update 14, 5 (2004), 15-20.

[2] CEN-CENELEC-ETSI Smart Grid Coordination Group. 2012. Smart Grid Architecture Model. https://www.cencenelec .eu/standards/Sectors/SustainableEnergy/SmartGrids/Pages/default .aspx

[3] Ching Huei Chen. 2014. An adaptive scaffolding e-learning system for middle school students' physics learning. Australasian fournal of Educational Technology 30, 3 (Aug. 2014). https://www.learntechlib .org/p/148483/

[4] Nada Dabbagh. 2005. Pedagogical models for E-Learning: A theorybased design framework. In International fournal of Technology in Teaching and Learning. 25-44.

[5] Marijana Despotović-Zrakić, Konstantin Simić, Aleksandra Labus, Aleksandar Milić, and Branislav Jovanić. 2013. Scaffolding Environment for Adaptive E-learning through Cloud Computing. Journal of Educational Technology \& Society 16, 3 (July 2013), 301-314. http://www.jstor.org/stable/jeductechsoci.16.3.301

[6] DIN Deutsches Institut für Normung e. V. 2016. Reference Architecture Model Industrie 4.0 (RAMI4.0). Technical Report. Berlin. https://www .beuth.de/en/technical-rule/din-spec-91345-en/250940128

[7] Roland Heidel, Michael Hoffmeister, Martin Hankel, and Udo Döbrich 2017. Industrie4.0 Basiswissen RAMI4.0: Referenzarchitekturmodell mit Industrie4.0-Komponente. VDE Verlag $\mathrm{GmbH}$, Berlin Offenbach https://www.beuth.de/de/publikation/ram-industrie-40/253488526

[8] Cindy E. Hmelo-Silver and Howard S. Barrows. 2006. Goals and Strategies of a Problem-based Learning Facilitator. Interdisciplinary fournal of Problem-Based Learning 1, 1, Article 4 (May 2006). https://doi.org/ 10.7771/1541-5015.1004

[9] Cindy E. Hmelo-Silver, Ravit Golan Duncan, and Clark A. Chinn. 2007. Scaffolding and Achievement in Problem-Based and Inquiry Learning: A Response to Kirschner, Sweller, and Clark (2006). Educational Psychologist 42, 2 (April 2007), 99-107. https://doi.org/10.1080/ 00461520701263368
[10] Nasser Jazdi. 2014. Cyber physical systems in the context of Industry 4.0. In 2014 IEEE International Conference on Automation, Quality and Testing, Robotics. IEEE, Cluj-Napoca, Romania, 1-4. https://doi.org/ 10.1109/AQTR.2014.6857843

[11] Claudia Kaar, Josef Frysak, Christian Stary, Udo Kannengiesser, and Harald Müller. 2018. Resilient Ontology Support Facilitating MultiPerspective Process Integration in Industry 4.0. ACM Press, 1-10. https://doi.org/10.1145/3178248.3178253

[12] Henning Kagermann, Wolfgang Wahlster, and Joachim Helbig. 2013. Recommendations for implementing the strategic initiative INDUSTRIE 4.0. Technical Report. Frankfurt am Main.

[13] Marcia C. Linn, Elizabeth A. Davis, and Philip Bell. 2004. Internet environments for science education. Lawrence Erlbaum Associates Publishers, Mahwah, NJ, US.

[14] Felix Mayer, Dorothea Pantförder, Christian Diedrich, and Birgit Vogel-Heuser. 2013. Deutschlandweiter I4.0-Demonstrator - Technisches Konzept und Implementierung. Technical Report. Lehrstuhl für Automatisierung und Informationssysteme. https://mediatum.ub.tum .de/doc/1178726/1178726.pdf

[15] Plattform Industrie 4.0. [n. d.]. Agentenbasierte Vernetzung von CyberPhysischen Produktionssystemen (CPPS). http://www.plattform-i 40.de/I40/Redaktion/DE/Anwendungsbeispiele/265-agentenbasierte -vernetzung-von-cyber-physischen-produktionssystemen-tu -muenchen/agentenbasierte-vernetzung-von-cyber-physischen -produktionssystemen.html

[16] Plattform Industrie 4.0. 2016. Implementation Strategy Industrie 4.0: Report on the results of the Industrie 4.0 Platform. Leitfaden. Bitkom, VDMA, ZVEI, Berlin, Heidelberg. 96 pages. https://www.bitkom .org/Bitkom/Publikationen/Implementation-Strategy-Industrie -40-Report-on-the-results- of-the-Industrie-40-Platform.html

[17] Chris Quintana, Brian J. Reiser, Elizabeth A. Davis, Joseph Krajcik, Eric Fretz, Ravit Golan Duncan, Eleni Kyza, Daniel Edelson, and Elliot Soloway. 2004. A Scaffolding Design Framework for Software to Support Science Inquiry. Fournal of the Learning Sciences 13, 3 (July 2004), 337-386. https://doi.org/10.1207/s15327809jls1303_4

[18] Brian J. Reiser. 2004. Scaffolding Complex Learning: The Mechanisms of Structuring and Problematizing Student Work. Fournal of the Learning Sciences 13, 3 (July 2004), 273-304. https://doi.org/10.1207/s 15327809jls1303_2

[19] Jochen Schlick, Peter Stephan, Matthias Loskyll, and Dennis Lappe. 2014. Industrie 4.0 in der praktischen Anwendung. In Industrie 4.0 in Produktion, Automatisierung und Logistik: Anwendung Âủ Technologien Ẩ Migration, Thomas Bauernhansl, Michael ten Hompel, and Birgit Vogel-Heuser (Eds.). Springer Fachmedien Wiesbaden, Wiesbaden, 57-84. https://doi.org/10.1007/978-3-658-04682-8_3

[20] VDI/VDE-Gesellschaft Mess- und Automatisierungstechnik. 2014. Industrie 4.0 - Gegenstände, Entitäten, Komponenten. VDIStatusreport. https://www.vdi.de/presse/publikationen/publikationen -details/pubid/industrie-40-gegenstaende-entitaeten-komponenten/

[21] VDI/VDE-Gesellschaft Mess- und Automatisierungstechnik. 2015. Reference Architecture Model Industrie 4.0 (RAMI4.0). Technical Report. VDI/VDE.Gesellschaft Mess- und Automatisierungstechnik. https://www.vdi.de/presse/publikationen/publikationen -details/pubid/reference-architecture-model-industrie-40-rami 40-english-version/

[22] Lev S. Vygotskij. 1978. Mind in Society: The Development of Higher Psychological Processes. Presidential and Fellows of Harvard College.

[23] David Wood, Jerome S. Bruner, and Gail Ross. 1976. The Role of Tutoring in Problem Solving. Journal of Child Psychology and Psychiatry 17, 2 (April 1976), 89-100. https://doi.org/10.1111/j.1469-7610.1976.tb 00381.x 